\title{
DOES PATHOLOGICAL T3A UPSTAGING OF CLINICAL T1 STAGE HAS ANY DIFFERENCE ON LONG-TERM SURVIVAL WHEN COMPARED TO PATHOLOGICAL AND CLINICAL T1 STAGE RENAL CELL CARCINOMA
}

\author{
Nouman Khan, M. Arshad Irshad Khalil, Azfar Ali, Aleeshba Naeem, Abdul Rahman, \\ Khurram Mir \\ Department of Surgical Oncology, Shaukat Khanum Memorial Cancer Hospital and Research Centre, Lahore, \\ Pakistan \\ Received: 21 February 2018 / Accepted: 25 June 2018
}

\begin{abstract}
Background: A high number of clinical T1 (cT1) stage renal cell carcinoma (RCC) is upstaged to pathological T3a (pT3a) stage on histopathological findings. Several study results show that there is no survival difference among those cT1 stage who are upstaged on histopathological findings to those who remain pT1 stage RCC.

Objectives: The objectives of this study were to assess any survival difference for cT1 stage renal cell carcinoma (RCC) which is upstaged to pT3a stage as compared to those which remain pT1 stage RCC on histopathological findings.

Materials and Methods: It was a retrospective cohort study looking at patient aged $\geq 18$ years with cT1 RCC who underwent nephrectomy between January 2006 and December 2016. Patients were divided into two groups based on histopathological findings (pT1 vs. pT3a). Survival was analysed for the two groups using Kaplan-Meier method, and the difference in survival was calculated using log-rank model.

Results: The study included 187 patients. The mean age at presentation was 52.56 years, with $58.3 \%$ of the patients being male while $41.7 \%$ were female. The most common presentation was incidental diagnosis $(50.3 \%)$. Overall 5 -year survival for cT1a and pT1a RCC was $68 \%$ while that for cT1a and pT3a RCC was $100 \%$. There was no significant survival difference among the two groups $(P=0.316)$. The overall 5 -year survival for $\mathrm{cT} 1 \mathrm{~b}$ and $\mathrm{pT} 1 \mathrm{~b}$ RCC was $81 \%$ while that for $\mathrm{cT} 1 \mathrm{~b}$ and $\mathrm{pT} 3 \mathrm{a}$ was $65 \%$. There was no significant survival difference among the two groups $(P=0.136)$.
\end{abstract}

Conclusion: We found no survival difference in $\mathrm{cT} 1 \mathrm{RCC}$ who were upstaged to pT3a on histopathology as compared to cT1 RCC-staged pT1 on histopathology.

Key words: Clinical T1 stage, pathological T3a stage, radical nephrectomy, renal cell carcinoma, survival

\section{Introduction}

According to the American Joint Committee on Cancer (AJCC) $8^{\text {th }}$ edition, ${ }^{[1]}$ clinical T1 (cT1) stage RCC is 7 or $<7 \mathrm{~cm}$ in size with no evidence of perinephric fat, renal sinus fat, pelvicalyceal system segmental or renal vessels or adrenal invasion on imaging modalities.

Correspondence: Dr. Nouman Khan, Department of Surgical

Oncology, Shaukat Khanum Memorial Cancer Hospital and Research

Centre, Lahore, Pakistan.

Email: dr_noumank@hotmail.com
Pathological T3a (pT3a) stage tumours are those tumours that have evidence of the involvement of perinephric fat or pelvicalyceal system or renal sinus fat or segmental/ renal vessels on pathological findings irrespective of tumour size. There is a significant survival difference among the various $\mathrm{pT}$ stage tumours and has been observed in multicentre cohort studies. ${ }^{[2,3]}$ According to several studies, there is no significant survival difference between the renal cell carcinomas that are clinically and pT1 stage when compared to cT1 stage tumours but pT3a tumours. ${ }^{[4,5]}$ The main aim of our study was to assess any 
survival difference between cT1 stage RCC which was upstaged to pT3a on histopathological examination.

\section{Materials and Methods}

This study involved a retrospective collection of data from January 2006 to December 2016 following approval from the Institutional Review Board of Shaukat Khanum Cancer Hospital and Research Centre, Lahore. Data included demographic features, clinical, pathological and oncological variables. Computed tomography/magnetic resonance imaging images and histopathological reports were reviewed, and the AJCC $8^{\text {th }}$ edition TNM staging system was used for clinical and pathological staging of the $\mathrm{RCC}$ retrospectively.

All those patients, who underwent surgery for renal tumours from January 2006 to December 2016 with proven histology of renal cell carcinoma having clinical stage T1 on initial diagnosis, were included in the study. Patients with histology other than RCC, nodal disease, metastatic disease or cT stage $>1$ were excluded from the study.

The patients were further divided into two groups. Group 1 included those patients who had cT1 stage disease on initial diagnosis and pT1 stage on histopathological findings. Group 2 included those patients who had cT1 stage but T3a histopathological stage. Data were analysed using SPSS 20 software, survival analysis was performed on the two groups using Kaplan-Meier method and any significant survival difference among the two groups was calculated using log rank model. $P<0.05$ was considered significant.

\section{Results}

A total of 187 patients were included in our study. The mean age at presentation was 52.56 years. There was a male preponderance $(n=109,58.3 \%)$, and $41.7 \%(n=78)$ were female patients. The most common presentation was incidental diagnosis $(50.3 \%), 30.5 \%$ of patients presented with pain and $18 \%$ of patients with haematuria. Other characteristics are summarised in Table 1.

Overall, 98 patients had cT1a stage tumour at presentation, among which 91 (92.9\%) had pT1a stage tumour and $7(7.1 \%)$ had pT3a on histopathological findings. Median survival for cT1a and pT1a was 108 months while that for cT1a and pT3a tumour was 132 months. Using Kaplan-Meier analysis, 5-year overall survival for cT1a and pT1a RCC was 68\% (standard error [SE]: 0.102) while that for cT1a and pT3a RCC was $100 \%$ [Figure 1]. There was no significant difference among the two groups in terms of overall survival using log-rank test $(P=0.316)$.

Similarly, 89 patients had T1b stage tumour at presentation, among which $76(85.4 \%)$ had pT1b stage tumour and $13(14.6 \%)$ patients had pT3a tumour. Median time of survival for cT1b and pT1b was 120 months and that for cT1b and pT3a stage tumour was 72.3 months as described in Table 2.

The overall 5-year survival for $\mathrm{cT} 1 \mathrm{~b}$ and $\mathrm{pT} 1 \mathrm{~b}$ RCC was $81 \%$ (SE: 0.062) while that for cT1b and pT3a was

\section{Table 1: Patient characteristics}

\begin{tabular}{l|c}
\hline Characteristics & $\boldsymbol{n}(\%)$ \\
\hline Male & $109(58.3)$ \\
\hline Female & $78(41.7)$ \\
\hline Right renal tumour & $93(49.7)$ \\
\hline Left renal tumour & $94(50.3)$ \\
\hline Pain at presentation & $57(30.5)$ \\
\hline Haematuria at presentation & $35(18)$ \\
\hline Diagnosed incidentally & $94(50.3)$ \\
\hline
\end{tabular}

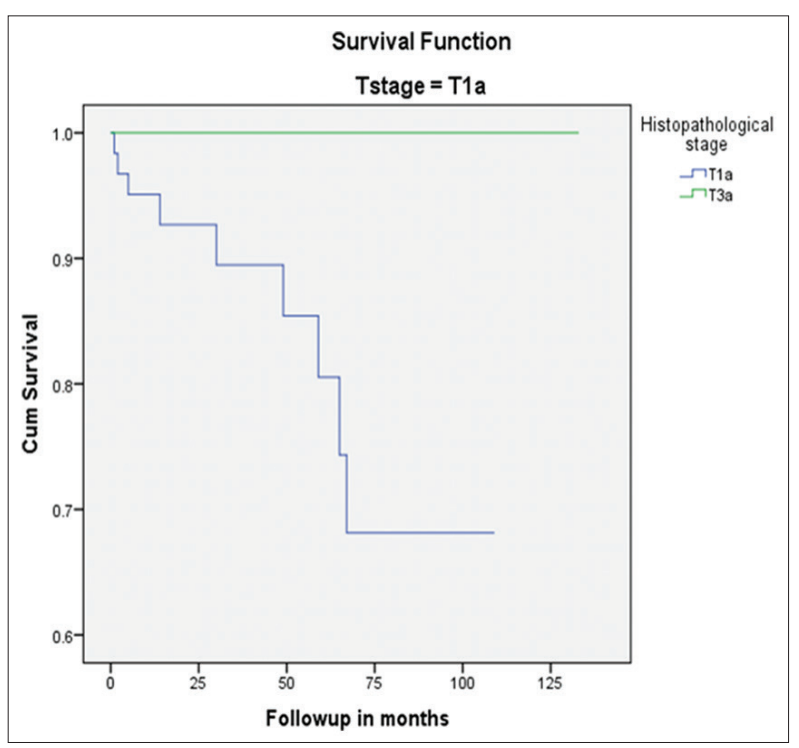

Figure 1: Survival function clinical T1 a versus pathological T3a 
Table 2: Median and overall survival

\begin{tabular}{l|c|c}
\hline Clinical and pathological T stage & Median survival (months) & Overall 5-year survival (\%) \\
\hline cT1a and pT1a & 108 & 68 \\
\hline cT1a and P T3a & 132 & 81 \\
\hline cT1b and pT1b & 120 & 65 \\
\hline cT1b and pT3a & 72.3 & 85 \\
\hline
\end{tabular}

cT1b: Clinical T1b, pT3a: Pathological T3a

$65 \%$ (SE: 0.17) using Kaplan-Meier model as shown in Figure 2. There was no significant survival difference among the two groups using log-rank test $(P=0.136)$. However, the projected difference in survival beyond 5 years needs to be further looked into.

Incidentally diagnosed patients had 5-year survival of $82 \%$ while those who presented with symptoms had 5-year survival of $72 \%$. There was no significant overall survival difference among the two groups $(P=0.119)$.

\section{Discussion}

We found that $7.1 \%$ and $14.6 \%$ of $\mathrm{cT} 1 \mathrm{a}$ and $\mathrm{cT} 1 \mathrm{~b}$ RCC are upstaged to pT3a on pathological evaluation, respectively. However, there was no significant difference in the median or overall survival of the two groups over 5 years.

Most of the renal cancers are diagnosed incidentally, ${ }^{[6,7]}$ and globally, the most common stage at presentations is cT $1{ }^{\left[{ }^{8]}\right.}$ Overall and disease-free survival of cT1 RCC is good and can be managed with radical nephrectomy, nephron-sparing surgery or active surveillance. ${ }^{[9]}$ As high as $30 \%$ of patients with cT1 stage tumour turn out to be pT3a stage tumour on histopathological findings. Since pT3a has an adverse survival outcome as compared to pT1 stage tumours, ${ }^{[2,3]}$ a high number of patients with cT1 stage are upgraded on final histopathological findings.

In our study, the upstaging was noted in $7.1 \%$ and $14.6 \%$ of cT1a and cT1b tumours, respectively, which was similar to other international studies $(5-15 \%) .^{[5,10]}$ The question, whether this upstaging of cT1 stage tumour on histopathological findings has any effect on survival outcomes, has been addressed in several studies and has concluded that there is no survival difference among the cT1 that is upstaged to pT3a as compared to pT1

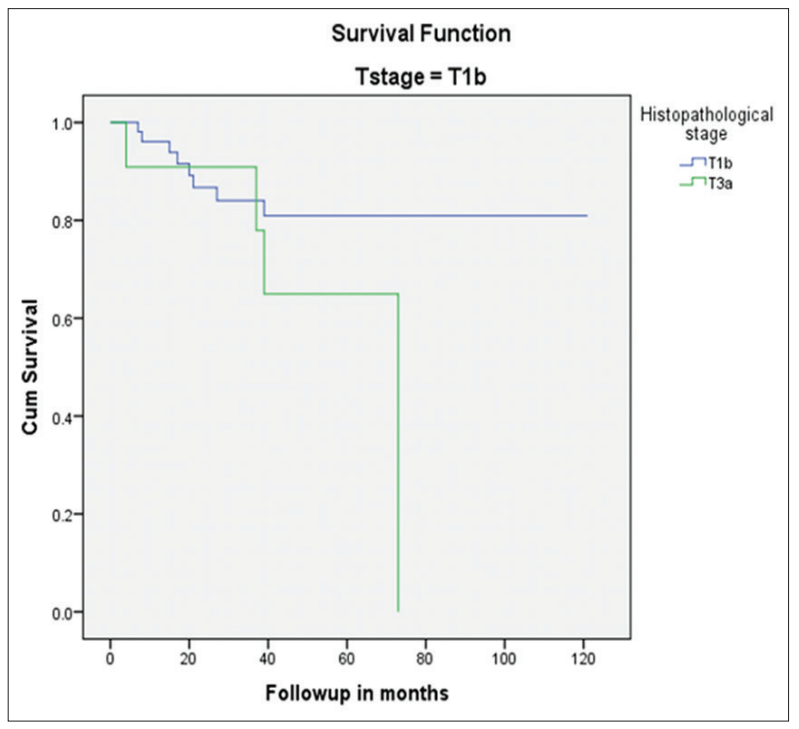

Figure 2: Survival function clinical T1b versus pathological T3a

stage tumours. ${ }^{[11,12]}$ We found that 5 -year overall survival for cT1a and pT1a RCC was $68 \pm 0.2 \%$ (SE: 0.102 , CI $95 \%$ ) while that for cT1a and pT3a RCC was $100 \%$. No significant difference was noted among the two groups in terms of overall survival using log-rank test $(P=0.316)$. Similarly, the overall 5-year survival for cT1b and pT1b RCC was $81 \pm 0.121 \%$ (SE: 0.062, confidence interval $95 \%$ ) while that for $\mathrm{cT} 1 \mathrm{~b}$ and $\mathrm{pT} 3 \mathrm{a}$ was $65 \%$, and there was no significant survival difference between the two groups $(P=0.136)$.

In our study, we noted $10.96 \%$ upstaging of cT1 stage renal cell cancer to pT3a stage and this upstaging is not associated with poor long-term outcomes. The number of patients in our study was much smaller, but our findings and literature review show similar results which demand further studies on this topic with a large number of patients. 


\section{Conclusion}

We found no survival difference in cT1 RCC who were upstaged to pT3a on histopathology as compared to $\mathrm{cT} 1$ RCC-staged pT1 on histopathology.

\section{Conflict of Interest}

The authors declare that they have no conflict of interest.

\section{References}

1. Robins DJ, Small AC, Amin MB, et al. The 2017 American joint committee on cancer eighth edition cancer staging manual: Changes in staging guidelines for cancers of the kidney, renal pelvis and ureter, bladder, and urethra. J Urol 2017; 197:1163.

2. Novara G, Ficarra V, Antonelli A, et al. Validation of the 2009 TNM version in a large multi-institutional cohort of patients treated for renal cell carcinoma: Are further improvements needed? Eur Urol 2010;58:588-95.

3. Kim SP, Alt AL, Weight CJ, et al. Independent validation of the 2010 American joint committee on cancer TNM classification for renal cell carcinoma: Results from a large, single institution cohort. J Urol 2011;185:2035-9.

4. Roberts WW, Bhayani SB, Allaf ME, et al. Pathological stage does not alter the prognosis for renal lesions determined to be stage $\mathrm{T} 1$ by computerized tomography. J Urol 2005;173:713-5.
5. Ramaswamy K, Kheterpal E, Pham H, et al. Significance of pathologic T3a upstaging in clinical T1 renal masses undergoing nephrectomy. Clin Genitourin Cancer 2015;13:344-9.

6. Hollingsworth JM, Miller DC, Daignault S, et al. Rising incidence of small renal masses: A need to reassess treatment effect. J Natl Cancer Inst 2006;98:1331-4.

7. Luciani LG, Cestari R, Tallarigo C. Incidental renal cell carcinoma-age and stage characterization and clinical implications: Study of 1092 patients (1982-1997). Urology 2000;56:58-62.

8. Laguna MP, Algaba F, Cadeddu J, et al. Current patterns of presentation and treatment of renal masses: A clinical research office of the endourological society prospective study. J Endourol 2014;28:861-70.

9. Ljungberg B, Bensalah $\mathrm{K}$, Canfield $\mathrm{S}$, et al. EAU guidelines on renal cell carcinoma: 2014 update. Eur Urol 2015;67:913-24.

10. Gorin MA, Ball MW, Pierorazio PM, et al. Outcomes and predictors of clinical T1 to pathological T3a tumor up-staging after robotic partial nephrectomy: A multiinstitutional analysis. J Urol 2013;190:1907-11.

11. Srivastava A, Patel HD, Joice GA, et al. Incidence of T3a up-staging and survival after partial nephrectomy: Sizestratified rates and implications for prognosis. Urol Oncol 2018;36:12.e7-000000.

12. Lee H, Lee M, Lee SE, et al. Outcomes of pathologic stage T3a renal cell carcinoma up-staged from small renal tumor: Emphasis on partial nephrectomy. BMC Cancer 2018;18:427. 